\title{
The Effects of Information Architecture and Atmosphere Style on the Usability of an Ecology Education Website
}

\author{
Chao-jen $\mathrm{Ku}^{1,2}$, Ji-Liang Doong ${ }^{2}$, and Li-Chieh Chen ${ }^{3}$ \\ ${ }^{1}$ Department of Visual Design, Hsuan Chuang University, \\ No. 48, Hsuan Chuang Rd. Hsinchu, Taiwan, 300 \\ ${ }^{2}$ Graduate Institute in Design Science, Tatung University, \\ No. 40, Chung Shan North Rd., Sec. 3, Taipei, Taiwan, 104 \\ ${ }^{3}$ Department of Media Design, Tatung University, \\ No. 40, Chung Shan North Rd., Sec. 3, Taipei, Taiwan \\ chaojenku@gmail.com, jldo@mail2000.com.tw, lcchen@ttu.edu.tw
}

\begin{abstract}
Ecology education is an important issue nowadays. But not everyone has equal opportunities to learn relevant topics through direct access to the great nature. In such a case, the platform of Web becomes the potential channel for people to learn ecological topics. Therefore, the key to success is to enhance the platform so that the performance is close to that of experiencing the great nature in person. In this study, expert interview was first conducted. The participants pointed out the differences in information architectures, atmosphere styles, the differences in learners' backgrounds were important factors needed to be considered while designing such a system. Therefore, several experiment websites were constructed based on different atmosphere styles. The results revealed that there were significant differences in browsing behaviors and the usability of websites between people from rural and urban. Websites with breadth architecture and natural atmosphere could reduce user's pressure and perceived workload.
\end{abstract}

Keywords: Ecology Education, World Wide Web, Information Architecture, Layout, Usability.

\section{Background and Motivation}

Ecological education is an important issue nowadays. But not everyone has equal opportunities to learn relevant topics through direct access to the great nature. In such a case, e-learning platform becomes the potential channel for those who are eager to learn ecological topics in classroom. How to enhance the platform so that the performance of e-learning in classroom is close to that of experiencing the great nature in person is the key to success. In addition, the differences in learners' growing-up environments are also important factors needed to be considered while constructing such a system. Therefore, the objective of this study is to examine whether different personal backgrounds or different webpage design styles and information architecture have significant influences on the performances of learning. 


\section{Literature Review}

\subsection{Information Architecture of Websites}

In the design factors of web site, the information architecture of web site directly affects the overall practicability of web site. Consequently, how to choose the appropriate link architecture of web pages is the challenge of the designers.

The link architecture of web pages is also called as topology. Some scholars have categorized the topology into six categories (Brinck et al., 2002): hierarchical or tree, linear or sequence, matrix or grid, full mesh, arbitrary network, and hybrid. The hierarchical is the most common architecture. Its advantage is more speedy browsing and easy to expand new information. The linear or sequence is appropriate for procedure, story, or relate to sequent information guiding browse. The matrix is good to reveal two-dimension space. The full mesh can link to all of other web pages from every page, therefore it can provide most speedy guiding browse. The designer can use arbitrary network if the information architecture is less organized. The hybrid architecture integrates more than two categories, and is the more common category in reality. When one website is designed for education, some researchers had the following findings. For the low prior knowledge learners, the results showed that the hierarchical structure supported better free recall performance and reduced feelings of disorientation. In contrast, the high prior knowledge learners performed better and followed more coherent reading sequences in the network structure (Amadieu et al., 2009).

\subsection{The Aesthetic and Emotional Aspects of Websites}

The aesthetic and emotional aspects of websites or e-learning systems have obtained much attention recently (Hartmann et al., 2007; Stenalt \& Godsk, 2006; Light, 2004). It was recognized that when the look and feel of an interface is pleasing, users are likely to be more tolerant, e.g. they may be more prepared to wait a few more seconds for a website to download (Sharp et al., 2007).

In addition, during the design process, thought should be given to maintaining a consistent expressive style from the top to the bottom of the page, showing its distinguishing features and giving users a unique impression (Nielsen, 2000). Such design styles and forms, including background, images, colors, interactive guide, data...etc., are able to combine the webpage's theme with the user's cognitive experiences, and aren't just a pursuit for new and popular elements, such as brilliant colors and dynamic changes.

The design that combines concepts of the natural ecology website with users' backgrounds should also consider the design style of simulated scenery, which is the presentation of true images and sounds from nature, allowing users to achieve better learning results.

\section{Study of Available Websites}

The method used for this study is based on considerations of the research purpose; literature is reviewed and the current webpage is analyzed. Further discussions on the webpage's architecture and design style, teaching method of ecology education, and 
learning achievements of media are carried out via interviews with three specialists and used as reference for modifying the interactive website's design. Afterwards, an experiment is designed using the new and old version web pages, in which the subjects are observed for reactions and habits when using the webpage, the subjects then fill in a questionnaire that is used for finding which version and users from which background achieved better learning results.

\subsection{Website Description}

Design style analysis of the original Taiwan natural ecology website: In the Government Information Office's natural ecology website (www.gio.gov.tw/info/ecology/ Chinese/), the homepage uses Flash and provides two options, one enters an audiovisual introduction based on text and the other enters the main menu. The main menu shows Taiwan's terrain and altitudes (Fig. 1).
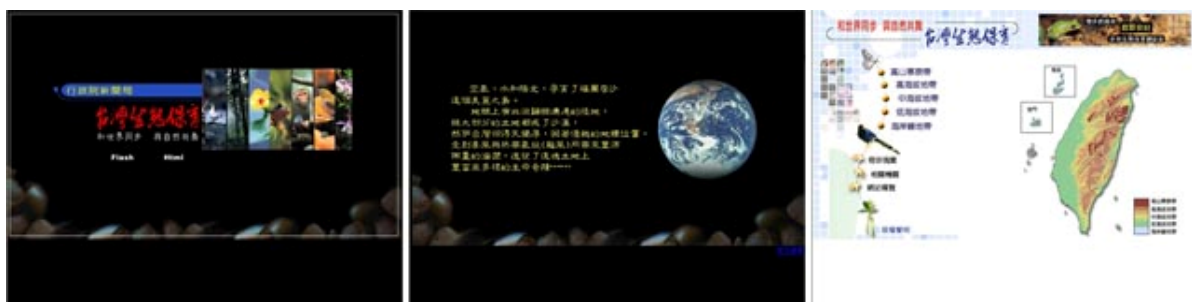

Fig. 1. The loading page and main page of the original ecology education website

When the mouse is over text on the main menu, users can understand Taiwan's terrain by watching the picture change to show which parts belong to the altitude the mouse is located over. Clicking on text or other items on the main menu will link to text descriptions of the terrain and ecology of the altitude, text links below the descriptions link to animals and plants within the altitude (Fig. 2).
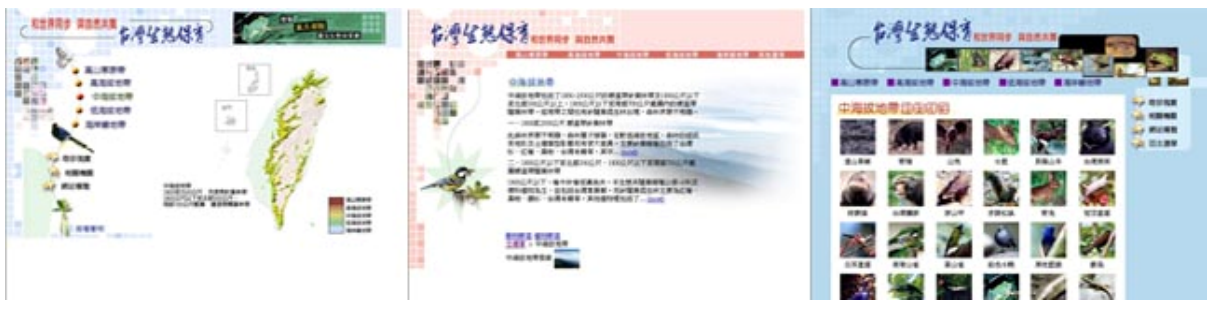

Fig. 2. The main page and the pages in the first two levels

After clicking on a specific species, a static image and text descriptions of the species' name, habitat, habitual behaviors...etc. are displayed. If you wish to hear the animal's sound, click on the speaker icon, or if you want to see a video of the animal, click on the camera icon and a new page will pop up to play the video (Fig. 3). The architecture of the website is illustrated in Figure 4. 


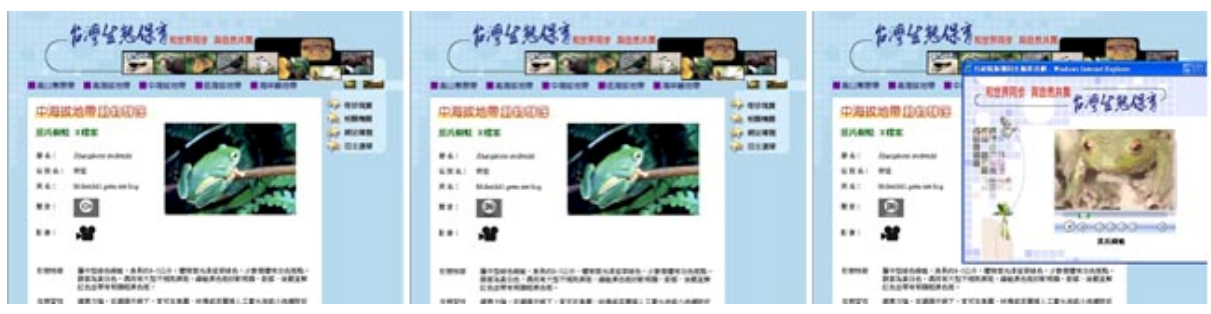

Fig. 3. The detailed pages of a specific creature

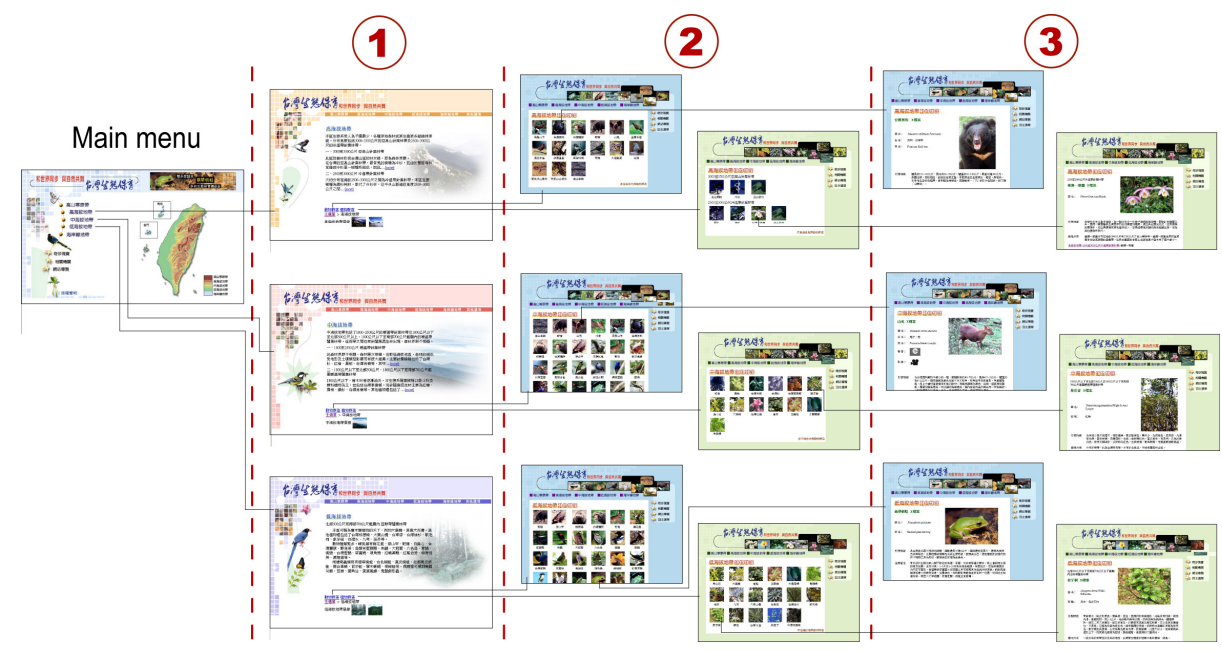

Fig. 4. The architecture of the original ecology education website

\subsection{Interviews with Specialists}

The opinions of three specialists, which are from different fields, on the current webpage's learning achievements are recorded on tape:

1. Web designer Chen Hsiu-chen: The architecture of websites is generally divided into Hierarchical and Network...etc.; hierarchical architecture has the advantage of clear categorization and easy to find data, but its disadvantages include causing the user to lose patience and learning capacity; network architecture has the advantage of rapidly finding targets, but will easily cause users to become lost. The interactive design of most learning websites are based on functional requirements; not many learning websites include aesthetic elements, simulate scenarios, combine text with images for easy search, and are integrated with databases. Such elements are more common in company websites, where the image and theme are emphasized on and visual and audio elements are well thought out.

2. High school biology teacher Chu Fang-lin: Students easily become tired of textbooks and explanations in class and hope to learn outside of school, such as zoos and botanical gardens, learning by seeing and hearing the real thing is very 
effective. In the computer classroom, websites are a very important tool for aiding teaching because they allow students to see recent news, such as fire ants, and learn related knowledge. Biology classes should be able to achieve even better learning results if a professional room and audiovisual equipment is provided to simulate scenarios for students to learn in.

3. Ecology photographer Pan Chien-hung: Tolerance and respect for the environment and nature can only result from the transfer of knowledge, as well as understanding and cognition. Ecology education is best presented in the form of videos, and will achieve the best results if shown on TV or in class using a DVD produced from a professional director, screenwriter, filming and editing (such as National Geographic and Discovery Animal Planet series).

The three specialists mentioned above were asked to use several existing natural ecology websites, they were observed and recorded by combining the Think Aloud method with screen capturing and sound recording, and their opinions are organized as follows: Taiwan's natural ecology websites have too much data, clicking on text to link to species' subkingdom, class, order, family, genus... requires too much professional knowledge, and the design emphasizes more on functionality and less on audiovisual and scenario creation, which makes searches difficult and prevents better learning achievements; dividing Taiwan into different altitudes is a better way for searching animals and plants.

\section{Experiment Design}

\subsection{The Atmosphere}

\subsubsection{The Design of a New Website with Natural Atmosphere}

Based on the results of literature review and the opinions of specialists, a 16:9 wide screen ratio is used to simulate a broader view, images and text information of the old version website are used a basic elements, and images and sounds of nature are enhanced to let users feel the atmosphere, becoming the website's unique style. The homepage uses a beautiful natural atmosphere to attract the user's attention, plays music and sounds of nature at the same time, and shows a menu that includes animals, plants, theme videos, and links to other websites.

After a species is selected, the image of a forest first appears, to the right is a diagram with text that indicates the altitude, semi-transparent animal images appear for each altitude, the normal colors of the animal appear when the mouse is moved over the image, and the animals name is shown to the bottom of the image.

The background turns dark, but the altitude diagram and animal images can still be used, text contents include the animal's name, scientific name, English name, characteristics, habitual behaviors and habitat.

The user can click on the speaker icon to listen to the animal's sound. When the camera icon is clicked on, the static animal image plays a dynamic video. The trangle arrow on the upper left corner allows the user to move back to the previous page until the main menu. 


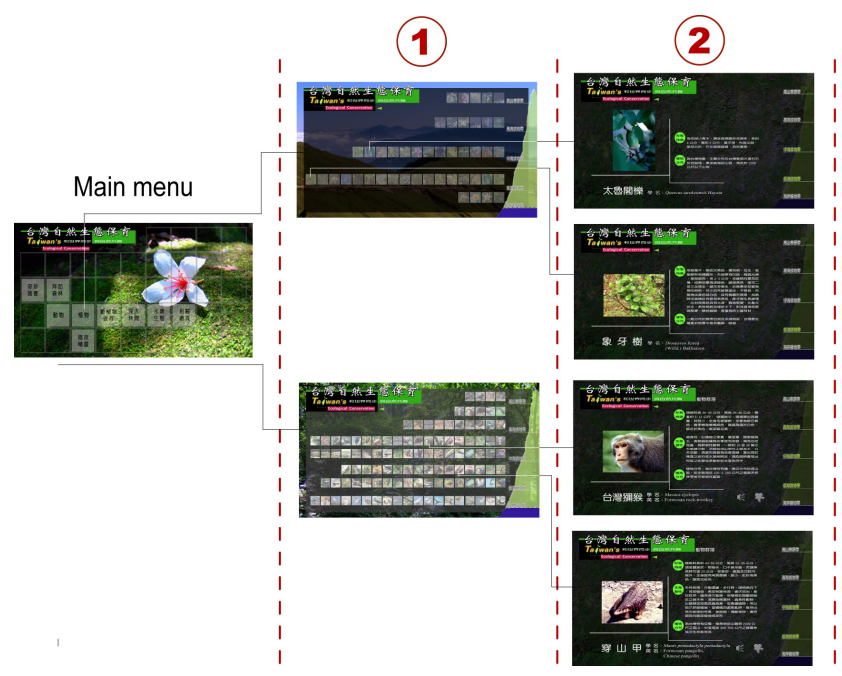

Fig. 5. The new website with breadth architecture and natural atmosphere

\subsubsection{Comparative Evaluation}

In the experiment, each participant was required to carry out typical tasks that interact with the interface prototypes. After that, the participant was asked to complete tests about the learning contents and system usability scale (SUS) and NASA-TLX questionnaire. The dependent variables in this research were the learners' performance, including the time consumed and the correctness of answering questions, and system usability evaluation.

The tasks are to search for pictures, text, sound effects and videos, and then draw them or compare them. Task one is to search for the shapes of leaves from two plants that grow on different altitudes: Ebony tree and Yellow water lily, and then draw them on the test sheet. Task two is to search for the Highland red-bellied swallowtail butterfly and write down its habitat and habitual behaviors. Task three is to compare the sounds of Formosan rock-monkey and Formosan reeve's muntjac. The screen capture software Cantasia is used to record how users operate the website, allowing the way users complete a task to be observed while comparing the accuracy of their answers. Subjects fill in a questionnaire with SUS and NASA-TLX on a 9-level Likert scale immediately after completing the tasks. Cross examination of positive and negative questions are used to find the subjects true subjective experience. The SUS part has 8 questions in total on easiness to learn, pictures and text, architecture and style. The NASA-TLX part has 6 questions in total on mental load, physical load, time load, energy consumption, performance and frustration.

Two groups of junior high school students participated in the experiments. Their growing-up environments were classified into "rural" and "urban." The urban group had 16 first-year students from Nei-Hu Junior High School in Taipei. They used World Wide Web frequently. The rural group had 16 first-year students from Bei-Nan Junior High School in the rural areas of Taitung. Internet access for them was not as convenient as that of the urban group. Therefore, they seldom used World Wide Web. 
Each group was further divided into two subgroups, one for experiencing the breadth -hierarchy sites, the other for the depth-hierarchy sites, respectively. The learning processes of the participants were observed and recorded through video and further converted into qualitative and quantitative data, such as browsing behaviors and time spent for learning.

\subsubsection{Results}

Test data were analyzed using two-way ANOVA. The results revealed that there were significant differences in browsing behaviors and the usability of websites between people from rural and urban. The students in the urban group tended to able to find the required information in the depth-hierarchy websites in shorter time. Students in the rural group tended to rely on the interface with natural atmosphere, and easily got frustrated in depth-hierarchy websites. Evidences showed that participants perceived less workload when using the website with natural atmosphere and situational music, no matter they were from rural or urban. In addition, evidences showed students using 16:9 wide-ratio webpage got better satisfaction then using the layout with traditional 4:3 ratio. Compared to traditional layout of websites, wide-screen layout provided better landscape view to simulate human's perception of the real world.

Table 1. The mean and standard deviation of task completion time

\begin{tabular}{lcc}
\hline Atmosphere & \multicolumn{2}{c}{ User Background } \\
\cline { 2 - 3 } & City & Rural \\
\hline Natural Atmosphere & $287.50(16.96)$ & $451.88(21.26)$ \\
Traditional Atmosphere & $384.50(19.61)$ & $424.25(20.60)$ \\
\hline
\end{tabular}

Table 2. The mean and standard deviation of user satisfaction

\begin{tabular}{lcc}
\hline Atmosphere & \multicolumn{2}{c}{ User Background } \\
\cline { 2 - 3 } & City & Rural \\
\hline Natural Atmosphere & $8.09(1.01)$ & $7.30(0.89)$ \\
Traditional Atmosphere & $6.72(1.21)$ & $6.83(1.61)$ \\
\hline
\end{tabular}

Table 3. The mean and standard deviation of rating in reducing workload

\begin{tabular}{lcc}
\hline Atmosphere & \multicolumn{2}{c}{ User Background } \\
\cline { 2 - 3 } & City & Rural \\
\hline Natural Atmosphere & $8.33(1.18)$ & $7.60(1.13)$ \\
Traditional Atmosphere & $6.94(1.08)$ & $6.46(1.04)$ \\
\hline
\end{tabular}

\subsection{The Information Architecture}

\subsubsection{The Modification of the Traditional Website}

In the second stage, two websites with same atmosphere but different information architectures were compared. The traditional website was modified by changing the 
screen ratio into 16:9 and by adding situational sounds from the great nature as those in the new website discussed in the previous section. The website with the depth architecture separates the menu of altitudes and the links to animals and plants navigation pages into two different pages. The website the breadth architecture combines the menu of altitudes and the navigation menus for animals and plants in the same page.

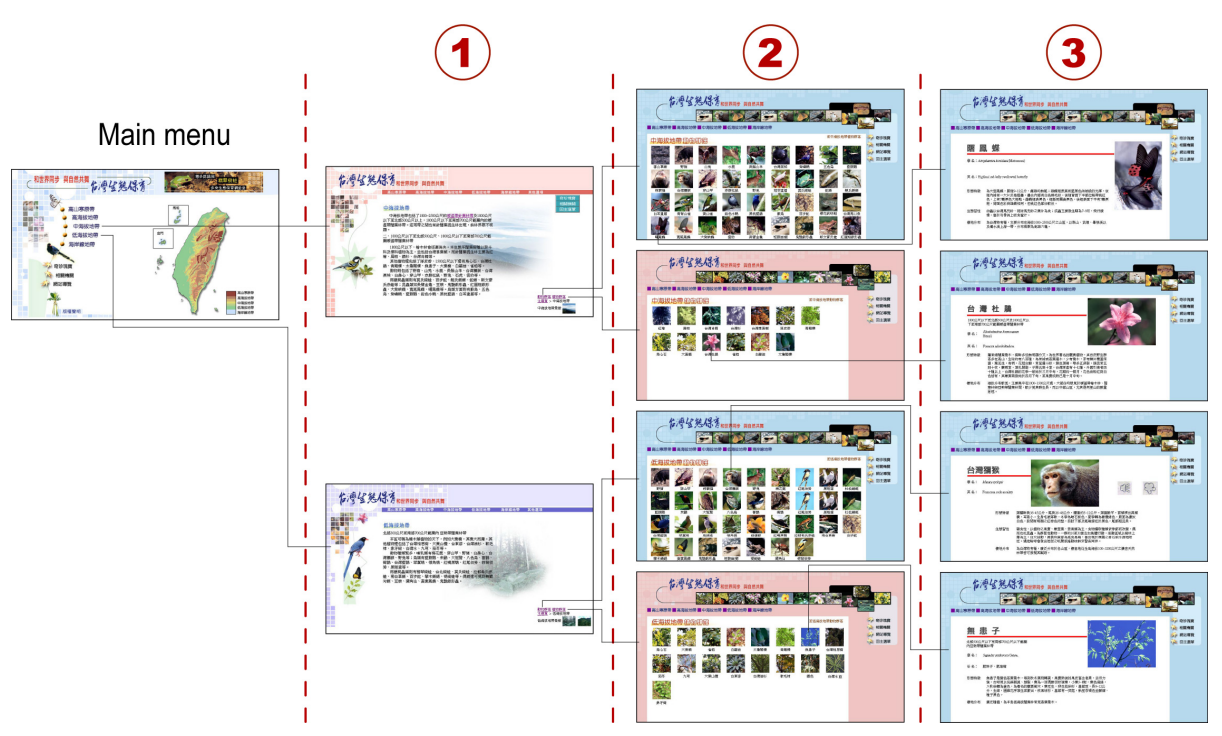

Fig. 6. The modified website with depth architecture and natural atmosphere

\subsubsection{Comparative Evaluation}

Twenty High School students participated in the experiments. They were divided into two groups. They carried out the same tasks as those in the first stage and answer the same set of questions in the post-test questionnaire.

\subsubsection{Results}

Test data were analyzed using t-test. The results showed that breath architecture is superior to depth architecture in reducing the steps of searching in ecology education websites.

Table 4. The mean and standard deviation of ratings

\begin{tabular}{lcc}
\hline Information Architecture & \multicolumn{2}{c}{ Ratings } \\
\cline { 2 - 3 } & Rating in User Satisfaction & $\begin{array}{c}\text { Rating in Reducing } \\
\text { Workload }\end{array}$ \\
\hline Depth Architecture & $6.16(0.97)$ & $6.30(1.08)$ \\
Breadth Architecture & $6.43(1.29)$ & $6.65(1.32)$ \\
\hline
\end{tabular}




\section{Discussion and Conclusion}

These results demonstrated that although websites with depth-hierarchy may be helpful for people who were familiar with state-of-the-art technologies while browsing, this feature did not contribute to reducing the workload of ecology learning. Websites with breadth architecture and natural atmosphere could reduce user's pressure and perceived workload.

\section{References}

1. Amadieu, F., Tricot, A., Marine, C.: Prior knowledge in learning from a non-linear electronic document: Disorientation and coherence of the reading sequences. Computers in Human Behavior 25, 381-388 (2009)

2. Brinck, T., Gergle, D., Wood, S.D.: Usability for the web. Morgan Kaufmann Publishers, San Francisco (2002)

3. Hartmann, J., Sutcliffe, A., Angeli, A.D.: Investigating attractiveness in web user interfaces. In: Proceedings of the SIGCHI conference on Human factors in computing systems, 2007, San Jose, California, USA, pp. 387-396 (2007)

4. Light, A.: Designing to persuade: the use of emotion in networked media. Interacting with Computers 16(4), 729-738 (2004)

5. Nielsen, J.: Designing Web Usability: The Practice of Simplicity. New Riders Publishing, Indiana (2000)

6. Sharp, H., Rogers, Y., Preece, J.: Interaction Design: Beyond Human-Computer Interaction, 2nd edn. Wiley, Chichester (2007)

7. Stenalt, M.H., Godsk, M.: The Pleasure of E-learning - Towards Aesthetic E-learning Platforms. In: Proceedings of the 12th International Conference of European University Information Systems, University of Tartu \& EUNIS 2006, Tartu, Estonia, June 2006, pp. 210 212 (2006) 